\title{
Erratum: "Visualization of single-walled carbon nanotubes electrical networks by scanning force microspy" [Appl. Phys. Lett. 79, 2979 (2001)]
}

Cite as: Appl. Phys. Lett. 80, 1103 (2002); https://doi.org/10.1063/1.1448652

Published Online: 01 February 2002

P. J. de Pablo, C. Gómez-Navarro, A. Gil, et al.

\section{ARTICLES YOU MAY BE INTERESTED IN}

Performing current versus voltage measurements of single-walled carbon nanotubes using scanning force microscopy

Applied Physics Letters 80, 1462 (2002); https://doi.org/10.1063/1.1453475

Erratum: "Performing current versus voltage measurements of single-walled carbon nanotubes using scanning force microscopy" [Appl. Phys. Lett. 80, 1462 (2002)]

Applied Physics Letters 80, 2421 (2002); https://doi.org/10.1063/1.1471915

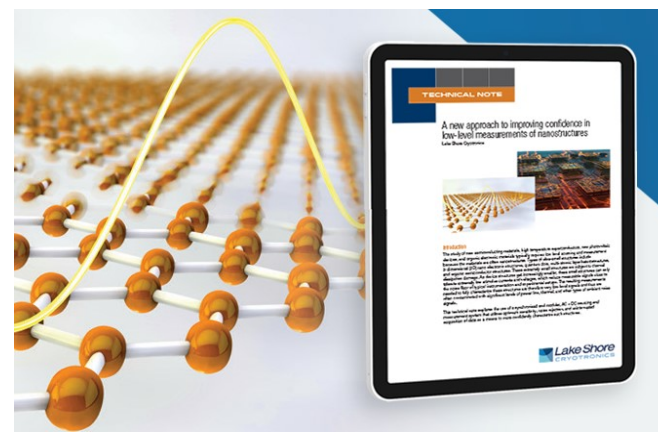

A new approach to low-level measurements of nanostructures Read our technical note

Download Now Lake Shore
CRYOTRONICS 


\section{ERRATUM}

\section{Erratum: "Visualization of single-walled carbon nanotubes electrical networks by scanning force microspy" [Appl. Phys. Lett. 79, 2979 (2001)]}

P. J. de Pablo, C. Gómez-Navarro, A. Gil, and J. Colchero

Laboratorio de Nuevas Microscopías, Departamento de Física de la Materia Condensada, Universidad Autónoma de Madrid, E-28049 Madrid, Spain

M. T. Martínez, A. M. Benito, and W. K. Maser

Instituto de Carboquímica, CSIC María de Luna 12, E-50015 Zaragoza, Spain

J. Gómez-Herrero and A. M. Baró

Laboratorio de Nuevas Microscopías, Departamento de Física de la Materia Condensada, Universidad Autónoma de Madrid, E-28049 Madrid, Spain

[DOI: $10.1063 / 1.1448652]$

The last word of the title should be "microscopy." The correct title should read: "Visualization of single-walled carbon nanotubes electrical networks by scanning force microscopy." 